\title{
Kostenübernahme einer Liposuktion bei Lipödem
}

\author{
Dr. Gwendolyn Gemke, Cosima Vossenkuhl - Rechtsanwältinnen, München
}

\author{
Endlich eine positive Entscheidung für alle Patientinnen mit \\ Lipödemen: erstmals wurde eine gesetzliche Krankenversiche- \\ rung dazu verurteilt, die Kosten der Liposuktion zu überneh- \\ men.
}

In medizinischen Fachkreisen ist das Lipödem längst als Krankheit anerkannt. Die gesetzlichen und privaten Krankenversicherer lehnen die Erstattung von Behandlungskosten dennoch in der Regel mit der Begründung ab, es handle sich um ein kosmetisches Problem. Den versicherten Patientinnen wird empfohlen, Sport zu treiben und ihr Gewicht zu reduzieren.

\section{Ursachen des Lipödems unbekannt}

Die Ursachen des fast ausschließlich bei Frauen auftretenden Lipödems sind unbekannt. Zur Behandlung der Symptome (Unterhautfettgewebsvermehrung, Ödemund Hämatombildung) stehen konservative und operative Verfahren zur Verfügung. Bei der komplexen physikalischen Entstauungstherapie (KPE) wird die Ödembildung durch Lymphdrainage, Kompression und Bewegungstherapie reduziert. Krankhaft vermehrtes Fettgewebe kann hierdurch jedoch nicht reduziert werden, sondern muss operativ im Rahmen einer Liposuktion entfernt werden. Die Kosten einer Liposuktion sind erheblich - eine Absaugung an beiden Beinen (Ober-, Unterschenkel und Knie beidseits) kostet rund 10.000 EUR. Für die betroffenen Patientinnen ist die Erstattung durch einen Kostenträger daher meist zwingend.

Der Gemeinsame Bundesausschuss der Ärzte und Krankenkassen (GBA) hat keine positive Empfehlung für eine Liposuktion bei Lipödemen ausgesprochen. Aus diesem Grunde hat das Bundessozialgericht im Jahr 2008 die Klage gegen eine gesetzliche Krankenversicherung abgewiesen und einen Ausnahmefall, welcher das Abweichen von den Empfehlungen des GBA rechtfertigen könnte, nicht gesehen. Die Sozialgerichtsbarkeit entschied in den vergangenen Jahren in Übereinstimmung mit der Rechtsprechung des Bundessozialgerichts und wies entsprechende Klagen ab. In einer aktuellen Entscheidung (März 2012) hat das Sozialgericht Chemnitz nun erstmals eine andere Rechtsauffassung vertreten und gelangte zu dem Ergebnis, dass der GBA die Liposuktion als Behandlungsmethode für ein Lipödem längst hätte prüfen müssen. Dies umso mehr, als es im System der vertragsärztlichen Versorgung keine gesicherte Behandlung für ein Lipödem gebe. Die beklagte Krankenversicherung hätte lediglich die konservative Therapie übernommen. Anhand des Krankheitsverlaufs war jedoch ersichtlich, dass dies die Erkrankung der Klägerin weder heilen noch eine Verschlimmerung verhüten konnte. Aus dem Ergebnis der Beweisaufnahme, insbesondere auf Basis der MDK-Gutachten sowie Sachverständigen-Gutachten, schloss das Gericht auf einen Systemmangel und verpflichtete die beklagte gesetzliche Krankenversicherung zur Kostenerstattung für eine Liposuktion beider Oberschenkel.

\section{Medizinisch notwendig?}

Im Bereich der privaten Krankenversicherung kommt es grundsätzlich auf die medizinische Notwendigkeit des Eingriffs an ( $\$ 1$ Absatz $2 \mathrm{MB} / \mathrm{KK})$. In aktuellen Urteilen (Amtsgericht Recklinghausen 2011, Landgericht Dortmund 2008) hat die Rechtsprechung hierüber stets nach sachverständiger Beratung im Rahmen der Beweisaufnahme entschieden, wobei in fast allen Fällen die private Krankenversicherung zur Kostenerstattung verpflichtet wurde.

Im Ergebnis ist festzustellen, dass die Durchführung einer Liposuktion bei Lipödemen aufgrund der erheblichen Kosten in der Regel von der Kostenübernahme durch die gesetzliche oder private Krankenversicherung abhängen wird. Nach neuer Rechtsprechung besteht nun in beiden Bereichen eine gute Chance, die Ansprüche der betroffenen Patientin durchzusetzen, sofern die entscheidenden Voraussetzungen erfüllt und richtig vorgetragen werden.

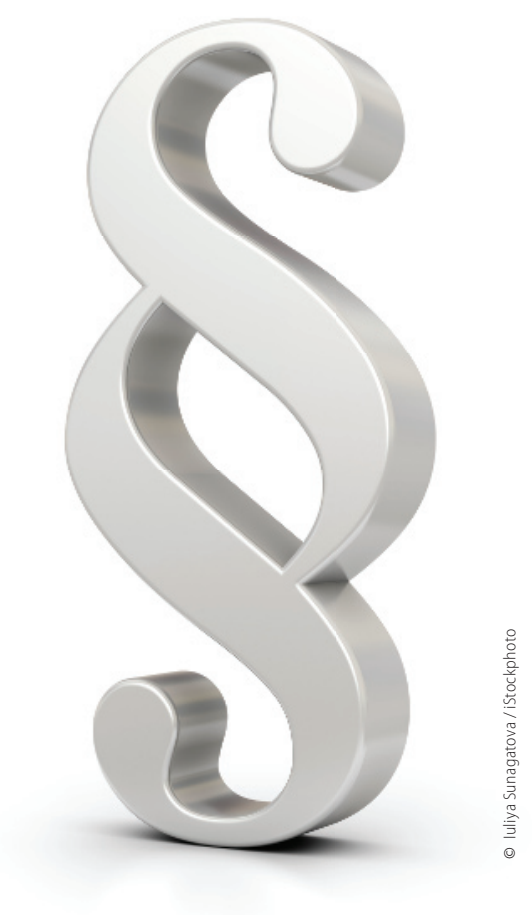

Künftig bleibt zu hoffen, dass sich weitere Gerichte der Auffassung des Sozialgerichts Chemnitz anschließen und einen Systemmangel bejahen werden. Darüber hinaus ist damit $\mathrm{zu}$ rechnen, dass sich der GBA nun in absehbarer Zeit mit dieser Problematik auseinandersetzen wird - dies wurde vom Vorsitzenden des GBA bereits angekündigt.

\section{Kostenübernahme früh abklären!}

Der behandelnde Arzt sollte betroffenen Patientinnen dazu raten, die Frage der Kostenübernahme mit der Krankenversicherung frühzeitig abzuklären. Befundstatus und Krankheitsverlauf müssen genau dokumentiert werden, damit die relevanten Punkte im Rahmen einer Erstattungsklage dargestellt und nachgewiesen werden können. Gegebenenfalls sind zur Erleichterung der Durchsetzbarkeit auch eine Fotodokumentation und die Einholung einer zweiten ärztlichen Meinung erforderlich. Sofern sich Schwierigkeiten seitens der Krankenversicherung abzeichnen, sollte den Patientinnen die rechtzeitige Einholung anwaltlicher Beratung empfohlen werden. 